\title{
Universal dynamics of superradiant phase transition in the anisotropic quantum Rabi model
}

\author{
Xunda Jiang ${ }^{1,2}$, Bo Lu ${ }^{1}$, Chengyin Han ${ }^{1}$, Ruihuan Fang ${ }^{1,2}$, \\ Minhua Zhao ${ }^{1,2}$, Zhu Ma ${ }^{1,2}$, Tian Guo ${ }^{1,2}$, and Chaohong Lee ${ }^{1,2}$ * \\ ${ }^{1}$ Guangdong Provincial Key Laboratory of Quantum Metrology and Sensing \& School of Physics and Astronomy, \\ Sun Yat-Sen University (Zhuhai Campus), Zhuhai 519082, China and \\ ${ }^{2}$ State Key Laboratory of Optoelectronic Materials and Technologies, \\ Sun Yat-Sen University (Guangzhou Campus), Guangzhou 510275, China
}

\begin{abstract}
We investigate the universally non-equilibrium dynamics of superradiant phase transition in the anisotropic quantum Rabi model. By introducing position and momentum operators, we obtain the ground states and their excitation gaps for both normal and superradiant phases via perturbation theory. We analytically extract the critical exponents from the excitation gap and the diverging length scale near the critical point, and find that the critical exponents are independent upon the anisotropy ratio. Moreover, by simulating the real-time dynamics across the critical point, we numerically extract the critical exponents from the phase transition delay and the diverging length scale, which are well consistent with the analytical ones. Our study provides a dynamic way to explore universal critical behaviors in the quantum Rabi model.
\end{abstract}

\section{INTRODUCTION}

Spontaneous symmetry breaking and quantum phase transitions (QPTs) are two fundamental and important concepts in physics. The second-order QPTs always associate with spontaneous symmetry breaking [1 3], in which gapless energy spectra and degenerate ground states appear in the thermodynamical limit. Due to the gapless excitations at the critical point, the adiabaticity breaks down when a system goes through a continuous phase transition. As a consequence, nontrivial excitations such as domains [4 14], vortices 15 17] and solitons [18 20 appear spontaneously and obey the wellkonwn Kibble-Zurek mechanism (KZM) 3, 4, 21 25]. The KZM has been extensively studied in various systems, from the early universe 3, 4], condensed matter systems 26 28, trapped ions 29 33], to ultracold atomic gases [5 15, 18 20, 34 37].

The quantum Rabi model (QRM), a paradigmatic model in quantum optics, describes the fundamental interaction between quantized fields and two-level quantum systems [38 44]. In the thermodynamic limit, the QRM exhibits normal-superradiant phase transition, which provides an excellent platform for exploring universal behavior in both equilibrium 45 48 and non-equilibrium dynamics [49 51]. The anisotropic QRM, whose rotating and counter-rotating interactions have different coupling strengths [52 54], is a generalized QRM. In recent, QPTs in the anisotropic QRM and their universality are studied [55]. However, the corresponding non-equilibrium universal dynamics is still unclear, it is worthy to clarify whether the anisotropic ratio affects the universality.

In this work, we investigated the non-equilibrium universal dynamics in the anisotropic QRM. Under the description of position and momentum operators, making

* lichaoh2@mail.sysu.edu.cn; chleecn@gmail.com use of the Schrieffer-Wolff (SW) transformation, we obtain the ground states and their excitation gaps with the second-order perturbation theory. Then, we analytically extract the critical exponents from the excitation gap and the diverging length scale, which reveal that the anisotropic QRM shares the same critical exponents for different anisotropy ratios between the rotating and counter-rotating terms. Furthermore, we numerically simulate the real-time dynamics of the anisotropic QRM whose coupling strength is linearly swept across the critical point. With the non-equilibrium dynamics, we numerically extract two universal scalings from the phase transition delay and the diverging length scale with respect to the quench time. The critical exponents extracted from the numerical simulation are well consistent with the analytical ones.

The paper is organized as follows. In Sec. II, we introduce the anisotropic QRM and give its ground states and excitation gaps. In Sec. III, we briefly review the KZM, and analytically extract the critical exponents from the excitation gap and the variance of the position and momentum operators. In Sec. IV, we present the real-time non-equilibrium universal dynamics, and extract the critical exponents from the phase transition delay and the diverging length scale. Finally, we give a brief summary and discussion in Sec. V.

\section{THE ANISOTROPIC QUANTUM RABI MODEL: GROUND STATES AND EXCITATION GAPS}

In the units of $\hbar=1$, the anisotropic QRM can be described by the full-quantum Hamiltonian,

$$
H=\omega a^{\dagger} a+\frac{\Omega}{2} \sigma_{x}+g\left[\left(\sigma_{+} a+\sigma_{-} a^{\dagger}\right)+\lambda\left(\sigma_{+} a^{\dagger}+\sigma_{-} a\right)\right],
$$


where $a^{\dagger}(a)$ are the creation (annihilation) operators of the phonons with frequency $\omega, g$ is the coupling strength and $\lambda$ denotes the anisotropic ratio between rotating and counter-rotating terms. Given the Pauli matrices $\sigma_{x, y, z}$, the second term describes a two-level system $\sigma_{ \pm}=\left(\sigma_{z} \mp i \sigma_{y}\right) / 2$ with a transition frequency $\Omega$.

Defining the dimensionless position and momentum operators $x=\left(a+a^{\dagger}\right) / \sqrt{2}$ and $p=i\left(a^{\dagger}-a\right) / \sqrt{2}$, the Hamiltonian reads

$H=\frac{\omega}{2}\left(p^{2}+x^{2}\right)+\frac{\Omega}{2} \sigma_{x}+\tilde{g} \sqrt{\frac{\Omega \omega}{8}}\left[(1+\lambda) \sigma_{z} x+(1-\lambda) \sigma_{y} p\right]$,

where $\tilde{g}=2 g / \sqrt{\Omega \omega}$. The Hamiltonian becomes the QRM when $\lambda=1$, while it is the JC model when $\lambda=0$. The second term becomes dominant in the limit $\Omega / \omega \rightarrow \infty$, thus the relevant low-energy states have $\left\langle\sigma_{x}\right\rangle \simeq-1$. Within this subspace, the ground states can be determined by the competition between the first term (a conventional oscillator) and the last term (the coupling between the phonon field and the two-level system) 55, 56. In Fig. 1. we show the typical ground state of Eq. (2) for different coupling strength. It clearly show the ground states undergoes a spontaneous symmetry breaking from symmetric to asymmetric when $\tilde{g}$ increases, see Fig. 1(a).
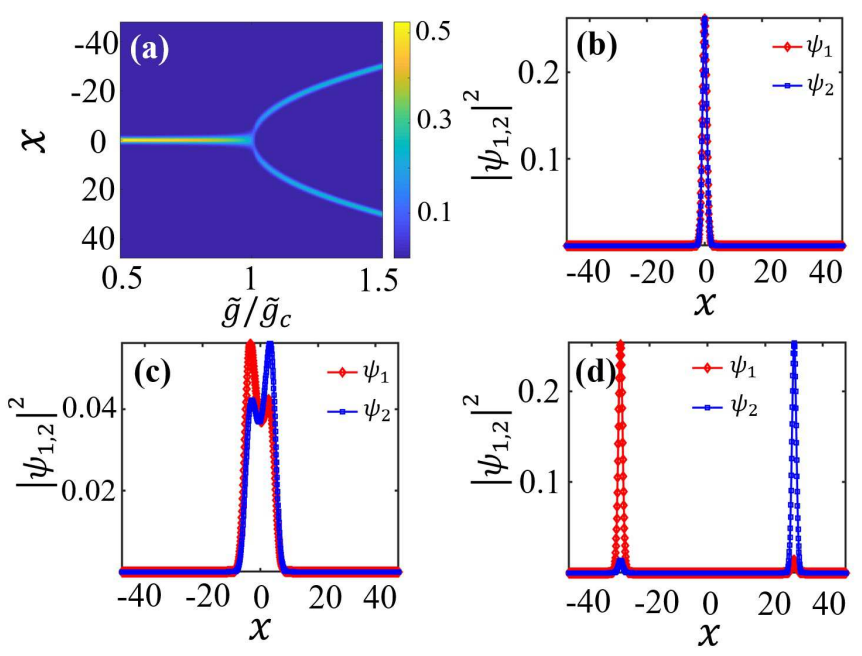

FIG. 1. (Color online) Density distributions of the ground states of the quantum Rabi model. (a) The total density distribution $\left|\psi_{1}\right|^{2}+\left|\psi_{2}\right|^{2}$ for $\lambda=1, \omega=1, \Omega=1000$ and $L=$ 96; (b,c,d) are three typical density distribution for $\tilde{g} / \tilde{g}_{c}=$ $0.5,1.02$ and 1.5 , respectively. In which, $\tilde{g} / \tilde{g}_{c}=1$ is the critical point.

In the weak coupling region, $\tilde{g}<\tilde{g}_{c}$, the ground state is dominated by the oscillator term in the normal phase as shown in Fig. 1 (b), which is the vacuum of the phonon field and atom in the low energy space. However, as the field-matter coupling is increased to the deep strong coupling regime $\tilde{g}>\tilde{g}_{c}$, the ground state turns from the normal phase to the superradiant phase, in which both the atom and the phonon field become excited [see
Fig. 1(c,d)]. To describe the superradiant phase transition, one may choose the excitation of the atom and the phonon field can be served as an order parameter [47, 51].

\section{A. Normal phase}

Below we briefly review the derivation of ground states and their excitation gaps from a low-energy effective Hamiltonian [55]. To obtain the low-energy effective Hamiltonian, one may apply the SW transformation. The Hamiltonian (2) includes an unperturbed Hamiltonian $H_{0}$ and a off-diagonal perturbation $H_{V}$ as follows

$$
\begin{aligned}
& H_{0}=\frac{\omega}{2}\left(p^{2}+x^{2}\right)+\frac{\Omega}{2} \sigma_{x}, \\
& H_{V}=\tilde{g} \sqrt{\frac{\Omega \omega}{8}}\left[(1+\lambda) \sigma_{z} x+(1-\lambda) \sigma_{y} p\right] .
\end{aligned}
$$

By introducing a unitary operator $S_{0}$ [55], which is the generator of the SW transformation and is an antiHermitian operator,

$$
S_{0}=i \tilde{g} \sqrt{\frac{\omega}{8 \Omega}}\left[(1-\lambda) \sigma_{z} p-(1+\lambda) \sigma_{y} x\right] .
$$

Therefore the second-order low-energy effective Hamiltonian is given as

$$
H_{\text {eff }}^{(2)}=\left\langle-\left|H_{e f f}^{(2)}\right|-\right\rangle \simeq \frac{\omega}{2}\left(1-\xi^{\prime 2}\right) p^{2}+\frac{\omega}{2}\left(1-\xi^{2}\right) x^{2},
$$

where $| \pm\rangle$ are the eigenstates of $\sigma_{x}, \xi=\tilde{g}(1+\lambda) / 2$ and $\xi^{\prime}=\tilde{g}(1-\lambda) / 2$. In the weak coupling region, the effective Hamiltonian behaves as the conventional harmonic oscillator as shown in Fig. 1(b), which corresponds to the normal phase. The normal phase is the vacuum of atom and phonon excitations. The excitation gap is given as

$$
\varpi_{0}=\omega \sqrt{\left(1-\xi^{2}\right)\left(1-\xi^{\prime 2}\right)} .
$$

The normal-to-superradiant phase transition occurs at $\varpi_{0}=0$, which gives $\xi_{c}=1$ or $\xi_{c}^{\prime}=1$, that is,

$$
\tilde{g}_{c}=\frac{2}{1+|\lambda|} \text {. }
$$

In the region of $\tilde{g} \leq \tilde{g}_{c}$, the ground state is a normal phase $\psi_{0}\left(x, \alpha_{0}\right)=e^{-S_{0}} \phi_{0}\left(x, \alpha_{0}\right)|-\rangle$ with

$$
\phi_{0}\left(x, \alpha_{0}\right)=\frac{\sqrt{\alpha}_{0}}{\pi^{1 / 4}} \exp \left(-\frac{1}{2} \alpha_{0}^{2} x^{2}\right)
$$

denoting the ground state of the harmonic oscillator. Here the effective mass $m_{0}=1 /\left[\omega\left(1-\xi^{\prime 2}\right)\right]$ and the wavepacket width $\alpha_{0}=\sqrt{m_{0} \varpi_{0}}$.

\section{B. Superradiant phase}

We now discuss the ground states and the corresponding excitation gaps for the superradiant phase. In the 
region of $\tilde{g}>\tilde{g}_{c}$, the system enters into the superradiant phase and the effective Hamiltonian (5) for the normal phase breaks down. This means that $P=|-\rangle\langle-|$ is no longer the suitable low-energy subspace. Making use of the SW transformation, we introduce new generators to diagonalize the Hamiltonian for both $\lambda>0$ and $\lambda<0$. Then, one may obtain an effective Hamiltonian and give its ground states and excitation gaps.

In the case of $\lambda>0$, we introduce a new displaced operator $\mathcal{D}_{1}[\alpha]=e^{-i \alpha p}=e^{-\alpha \frac{\partial}{\partial x}}$ with the parameter $\alpha$ to be determined, thus the Hamiltonian (2) is transformed as

$$
\begin{aligned}
& H(\alpha)=\mathcal{D}_{1}^{\dagger}(\alpha) H \mathcal{D}_{1}(\alpha)=\frac{\omega}{2}\left(p^{2}+x^{2}\right)+\frac{\Omega}{2} \sigma_{x} \\
& +\sqrt{\frac{\Omega \omega}{2}}\left(\xi \sigma_{z} x+\xi^{\prime} \sigma_{y} p\right)+\omega \alpha x+\frac{\alpha \delta_{1}}{2} \sigma_{z}+\frac{\omega \alpha^{2}}{2}
\end{aligned}
$$

where $\delta_{1}=\sqrt{2 \Omega \omega} \xi$. The eigenstates of the atomic part $H_{a}=\frac{\Omega}{2} \sigma_{x}+\frac{\alpha \delta_{1}}{2} \sigma_{z}$ are

$|\uparrow\rangle=\cos \theta|+\rangle+\sin \theta|-\rangle, \quad|\downarrow\rangle=-\sin \theta|+\rangle+\cos \theta|-\rangle$,

with $\sin 2 \theta=\alpha \delta_{1} / \widetilde{\Omega}, \cos 2 \theta=\Omega / \widetilde{\Omega}$ and the new atomic transition frequency $\widetilde{\Omega}=\sqrt{\Omega^{2}+\left(\delta_{1} \alpha\right)^{2}}$. In terms of Pauli matrices $\tau_{x, y, z}$ associated with $\{|\uparrow\rangle,|\downarrow\rangle\}$, the Hamiltonian (9) becomes

$$
\begin{aligned}
H(\alpha)= & \frac{\omega}{2}\left(p^{2}+x^{2}\right)+\sqrt{\frac{\Omega \omega}{2}}\left(\xi \cos 2 \theta x \tau_{x}-\xi^{\prime} p \tau_{y}\right) \\
& +\left(\omega \alpha+\sqrt{\frac{\Omega \omega}{2}} \sin 2 \theta \xi \tau_{z}\right) x+\frac{\widetilde{\Omega}}{2} \tau_{z}+\frac{\omega \alpha^{2}}{2}
\end{aligned}
$$

To eliminate the perturbation term, $\left(\omega \alpha+\sqrt{\Omega \omega / 2} \sin 2 \theta \xi \tau_{z}\right) x$, we choose the parameter $\alpha$ such that $\omega \alpha-\sqrt{\Omega \omega / 2} \sin 2 \theta \xi=0$, which gives the nontrivial solutions

$$
\alpha= \pm \alpha_{g}= \pm \sqrt{\left(\Omega / 2 \omega \xi^{2}\right)\left(\xi^{4}-1\right)} .
$$

Given $\alpha= \pm \alpha_{g}$, the Hamiltonian reads

$$
\widetilde{H}\left( \pm \alpha_{g}\right)=\widetilde{H}_{0}+\widetilde{H}_{V}
$$

with

$$
\begin{aligned}
\widetilde{H}_{0} & =\frac{\omega}{2}\left(p^{2}+x^{2}\right)+\frac{\widetilde{\Omega}}{2} \tau_{z}, \\
\widetilde{H}_{V} & =\sqrt{\frac{\Omega \omega}{2}}\left(\xi \cos 2 \theta x \tau_{x}-\xi^{\prime} p \tau_{y}\right) .
\end{aligned}
$$

Making use of the SW transformation, we find a new generator,

$$
S_{1}=i \sqrt{\frac{\Omega \omega}{2 \widetilde{\Omega}^{2}}}\left(\xi^{\prime} p \tau_{x}+\xi \cos 2 \theta x \tau_{y}\right) .
$$

for diagonalizing the Hamiltonian (13) under the condition of $\tilde{\Omega} / \omega \gg 1$. Thus the second-order low-energy effective Hamiltonian reads,

$$
\widetilde{H}_{e f f}^{(2)} \simeq \frac{\omega}{2}\left(1-\frac{\Omega}{\widetilde{\Omega}} \xi^{\prime 2}\right) p^{2}+\frac{\omega}{2}\left(1-\frac{\Omega}{\widetilde{\Omega}} \xi^{2} \cos ^{2} 2 \theta\right) x^{2} .
$$

Comparing with the simple harmonic oscillator, the excitation gap (see Fig. 2) is given as

$$
\varpi_{1}=\omega \sqrt{\left(1-\frac{1}{\xi^{4}}\right)\left(1-\frac{\xi^{2}}{\xi^{2}}\right)} .
$$

The excitation gap recovers the previous result when $\lambda=$ 1 [51]. Obviously, the $\varpi_{1}$ vanishes at $\xi_{c}=1$, which gives the critical point

$$
\tilde{g}_{c}=\frac{2}{1+\lambda},(\lambda>0)
$$

The corresponding ground-state for $\tilde{g}>\tilde{g}_{c}$ is $\psi_{1}\left(x, \alpha_{1}\right)=$ $\mathcal{D}_{1}\left(\alpha_{g}\right) e^{-S_{1}} \phi_{0}\left(x, \alpha_{1}\right)|\downarrow\rangle$, where $\phi_{0}\left(x, \alpha_{1}\right)$ is the ground state of the harmonic oscillator with $\alpha_{1}=\sqrt{m_{1} \varpi_{1}}$ and the effective mass $m_{1}=1 /\left[\omega\left(1-\xi^{\prime 2} / \xi^{2}\right)\right]$.

In the case of $\lambda<0$, we introduce another displaced operator $\mathcal{D}_{2}(\beta)=e^{-i \beta x}=e^{-\beta \frac{\partial}{\partial p}}$ with the parameter $\beta$ to be determined, thus the Hamiltonian (2) is transformed as

$$
\begin{aligned}
& H(\beta)=\mathcal{D}_{2}^{\dagger}(\beta) H \mathcal{D}_{2}(\beta)=\frac{\omega}{2}\left(p^{2}+x^{2}\right)+\frac{\Omega}{2} \sigma_{x} \\
& +\sqrt{\frac{\Omega \omega}{2}}\left(\xi \sigma_{z} x+\xi^{\prime} \sigma_{y} p\right)+\omega \beta p+\frac{\beta \delta_{2}}{2} \sigma_{y}+\frac{\omega \beta^{2}}{2},
\end{aligned}
$$

where $\delta_{2}=\sqrt{2 \Omega \omega} \xi^{\prime}$. The eigenstates of the atomic part $H_{a}=\frac{\Omega}{2} \sigma_{x}+\frac{\beta \delta_{2}}{2} \sigma_{y}$ are

$$
|\widetilde{\uparrow}\rangle=\cos \theta^{\prime}|+\rangle-i \sin \theta^{\prime}|-\rangle,|\widetilde{\downarrow}\rangle=\sin \theta^{\prime}|+\rangle+i \cos \theta^{\prime}|-\rangle,
$$

with $\sin 2 \theta^{\prime}=\alpha \delta_{2} / \widetilde{\Omega}^{\prime}, \cos 2 \theta^{\prime}=\Omega / \widetilde{\Omega}^{\prime}$, and the new atomic transition frequency $\widetilde{\Omega}^{\prime}=\sqrt{\Omega^{2}+\left(\delta_{2} \beta\right)^{2}}$. In terms of Pauli matrices $\tau_{x, y, z}^{\prime}$ associated with $\{|\tilde{\uparrow}\rangle,|\tilde{\downarrow}\rangle\}$, the new Hamiltonian reads

$$
\begin{aligned}
H(\beta)= & \frac{\omega}{2}\left(p^{2}+x^{2}\right)-\sqrt{\frac{\Omega \omega}{2}}\left(\xi x \tau_{y}^{\prime}+\xi^{\prime} \cos 2 \theta p \tau_{x}^{\prime}\right) \\
& +\left(\omega \beta+\sqrt{\frac{\Omega \omega}{2}} \sin 2 \theta \xi^{\prime} \tau_{z}^{\prime}\right) p+\frac{\widetilde{\Omega}}{2} \tau_{z}^{\prime}+\frac{\omega \beta^{2}}{2} .
\end{aligned}
$$

To - eliminate $\left(\omega \beta+\sqrt{\Omega \omega / 2} \sin 2 \theta \xi^{\prime} \tau_{z}^{\prime}\right) p, \quad$ we choose $\omega \beta-$ $\sqrt{\Omega \omega / 2} \sin 2 \theta \xi^{\prime}=0$, which gives

$$
\beta= \pm \beta_{g}=\sqrt{\left(\Omega / 2 \omega \xi^{\prime 2}\right)\left(\xi^{4}-1\right)} .
$$

Given $\beta= \pm \beta_{g}$, the Hamiltonian becomes

$$
\widetilde{H}^{\prime}\left( \pm \beta_{g}\right)=\widetilde{H}_{0}^{\prime}+\widetilde{H}_{V}^{\prime}
$$

with

$\widetilde{H}_{0}^{\prime}=\frac{\omega}{2}\left(p^{2}+x^{2}\right)+\frac{\widetilde{\Omega}}{2} \tau_{z}^{\prime}, \widetilde{H}_{V}^{\prime}=-\sqrt{\frac{\Omega \omega}{2}}\left(\xi x \tau_{y}^{\prime}+\xi^{\prime} \cos 2 \theta p \tau_{x}^{\prime}\right)$. 
Through performing SW transformation, under the condition of $\tilde{\Omega}^{\prime} / \omega \gg 1$, we diagonalize the Hamiltonian (23) with the generator

$$
S_{2}=i \sqrt{\frac{\Omega \omega}{2 \widetilde{\Omega}^{2}}}\left(\xi x \tau_{x}^{\prime}-\xi^{\prime} \cos 2 \theta p \tau_{y}^{\prime}\right)
$$

Then we obtain the second-order low-energy effective Hamiltonian,

$$
{\widetilde{H^{\prime}}}_{\text {eff }}^{(2)} \simeq \frac{\omega}{2}\left(1-\frac{\Omega}{\widetilde{\Omega}} \xi^{\prime 2} \cos ^{2} 2 \theta\right) p^{2}+\frac{\omega}{2}\left(1-\frac{\Omega}{\widetilde{\Omega}} \xi^{2}\right) x^{2}
$$

and the excitation gap (see Fig. 2)

$$
\varpi_{2}=\omega \sqrt{\left(1-\frac{1}{\xi^{\prime}}\right)\left(1-\frac{\xi^{2}}{\xi^{\prime 2}}\right)}
$$

Obviously, the excitation gap $\varpi_{2}$ vanishes at the critical point $\xi_{c}^{\prime}=1$, that is,

$$
\tilde{g}_{c}=\frac{2}{1-\lambda},(\lambda<0)
$$

The corresponding ground state is $\psi_{2}\left(x, \alpha_{2}\right)=$ $\mathcal{D}_{2}\left(\beta_{g}\right) e^{-S_{2}} \phi_{0}\left(x, \alpha_{2}\right)|\tilde{\downarrow}\rangle$, where $\phi_{0}\left(x, \alpha_{2}\right)$ is the ground state of the simple harmonic oscillator with $\alpha_{2}=\sqrt{m_{2} \varpi_{2}}$ and the effective mass $m_{2}=1 /\left[\omega\left(1-1 / \xi^{\prime} 4\right)\right]$.

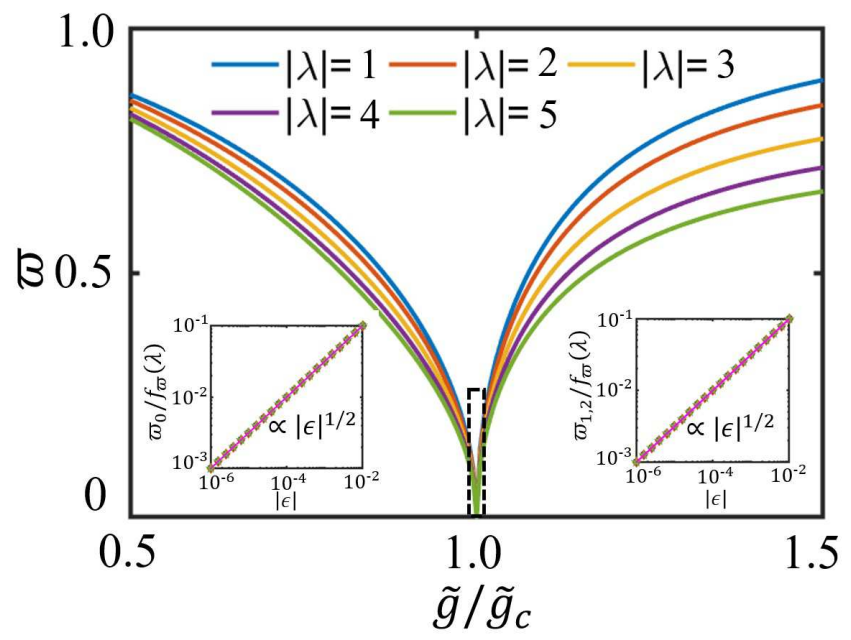

FIG. 2. (Color online) The excitation gap obtained from the second-order perturbative Hamiltonian. Insets: the universal scalings of the energy gaps near the critical region labelled by the dashed-line rectangle, where the left and right insets respectively correspond to normal and superradiant phases.

\section{UNIVERSAL CRITICAL DYNAMICS ACROSS SUPERRADIANT PHASE TRANSITION}

\section{A. Analytical Kibble-Zurek scalings}

In the following, we briefly introduce the KZM and analytically derive the universal critical exponents. Near the quantum critical point, due to the vanishing of the energy gap, the correlation (or healing) length $\zeta$ and relaxation time $\tau$ diverge as

$$
\tau \sim|\epsilon|^{-v z}, \quad \zeta \sim|\epsilon|^{-v}
$$

where $\epsilon$ is the dimensionless distance from the critical point, and $(v, z)$ are the critical exponents. To drive system from normal to superradiant phase, we linearly quench the coupling strength according to

$$
\epsilon(t)=\frac{\left|\tilde{g}(t)-\tilde{g}_{c}\right|}{\tilde{g}_{c}}=\frac{t}{\tau_{Q}},
$$

where $\tau_{Q}$ is the quench time. In a QPTs, the relaxation time is defined by the inverse of the gap between the ground state and the first relevant excited state, i.e. $\tau \simeq$ $\varpi^{-1}$. However, the relaxation time is divergent in the vicinity of the critical point, in which the gap vanishes as

$$
\varpi \sim|\epsilon|^{v z} .
$$

When the transition rate $|\dot{\epsilon} / \epsilon|=1 /|t|$ equals to the gap $\varpi \sim|\epsilon|^{v z}=\left|t / \tau_{Q}\right|^{v z}$, the adiabaticity fails near an instant $t=\hat{t}$,

$$
\hat{t} \sim \tau_{Q}^{\frac{v z}{1+v z}}, \quad \hat{\epsilon} \sim \tau_{Q}^{-\frac{1}{1+v z}}
$$

and the corresponding correlation length becomes as

$$
\hat{\zeta} \sim \hat{\epsilon}^{-v} \sim \tau_{Q}^{\frac{v}{1+v z}}
$$

In the region of $\tilde{g} \leq \tilde{g}_{c}$, the excitation gap $\varpi_{0}$ near the critical point vanishes as

$$
\varpi_{0} \propto f_{\varpi}(\lambda)|\epsilon|^{1 / 2}, \quad \lambda \neq 0,
$$

where

$$
f_{\varpi}(\lambda)=\omega\left[1-\left(\frac{1-|\lambda|}{1+|\lambda|}\right)^{2}\right]^{1 / 2}
$$

Comparing with Eq. 31, we analytically obtain $v z=$ $1 / 2$. When $\lambda=0$, the excitation gap becomes

$$
\varpi_{0} \propto|\epsilon|^{1} \text {. }
$$

Given $v z=1$, the critical exponents for $\lambda=0$ are belong to a different universality class [57], which will not be discussed below. For the anisotropic QRM, the energy gap $\varpi_{0}$ near the critical point vanishes, see the left insets 
of Fig. 2, it clearly reveals that the anisotropic QRM shares the same universal class.

To extract the critical exponents, we introduce the position variance $\Delta x$ and the momentum variance $\Delta p$. In the normal phase, $\Delta x$ and $\Delta p$ are obtained via the ground state $\psi_{0}$.

$$
\begin{aligned}
& \Delta x=\left[\frac{1}{2}\left(1-\frac{\omega}{\Omega} \xi \xi^{\prime}\right) \sqrt{\frac{1-\xi^{\prime 2}}{1-\xi^{2}}}+\frac{\omega \xi^{\prime 2}}{2 \Omega}\right]^{\frac{1}{2}}, \\
& \Delta p=\left[\frac{1}{2}\left(1-\frac{\omega}{\Omega} \xi^{\prime} \xi\right) \sqrt{\frac{1-\xi^{2}}{1-\xi^{\prime 2}}}+\frac{\omega \xi^{2}}{2 \Omega}\right]^{\frac{1}{2}} .
\end{aligned}
$$

Near the neighborhood of the phase transition, the length scale $\Delta x$ behaves as

$$
\begin{array}{ll}
\Delta x \propto f(\lambda)|\epsilon|^{-1 / 4}, & \lambda>0, \\
\Delta x \propto f^{-1}(\lambda)|\epsilon|^{1 / 4}, & \lambda<0,
\end{array}
$$

where

$$
f(\lambda)=\left[1-\left(\frac{1-|\lambda|}{1+|\lambda|}\right)^{2}\right]^{1 / 4}
$$

The critical behavior of $\Delta x$ shows that it is divergent when $\lambda>0$, while it vanishes when $\lambda<0$. It's worthy to note that $\Delta x$ plays an analogous role of the diverging length scale when $\lambda>0$ [1, 51]. Comparing with Eq. (29), we obtain the static correlation length critical exponent $v=1 / 4$ and the dynamic critical exponent $z=2$.

For the momentum variance $\Delta p$, its critical behavior obeys

$$
\begin{array}{ll}
\Delta p \propto f^{-1}(\lambda)|\epsilon|^{1 / 4}, & \lambda>0, \\
\Delta p \propto f(\lambda)|\epsilon|^{-1 / 4}, & \lambda<0 .
\end{array}
$$

In contrast to $\Delta x, \Delta p$ becomes divergent when $\lambda<0$, while it vanishes when $\lambda>0$. In the case of $\lambda<0$, the diverging length scale $\Delta p$ gives the critical exponent $v=1 / 4$ according to KZM, and so that we have the critical exponent $z=2$.

In the region of $\tilde{g}>\tilde{g}_{c}$, we divide the superradiant phase into two parts, which label as $x$-type( $p$-type) superradiant phase when $\lambda>0(\lambda<0)$ [55], respectively. In the superradiant phase, the excitation gap $\varpi_{1,2}$ near the critical point vanishes as

$$
\varpi_{1,2} \propto f_{\varpi}(\lambda)|\epsilon|^{1 / 2}, \quad \lambda \neq 0 .
$$

The critical behaviors of the excitation gap are shown in the right insets of Fig. 22 which gives $v z=1 / 2$. In the $x$-type superradiant phase, $\Delta x$ and $\Delta p$ are obtained via the ground state $\psi_{1}\left(x, \alpha_{1}\right)$.

$$
\Delta x=\left[\frac{1}{2}\left(1-\frac{\omega \xi^{\prime}}{\Omega \xi^{5}}\right) \sqrt{\frac{\xi^{2}-\xi^{\prime 2}}{\xi^{2}-\xi^{-2}}}+\frac{\omega \xi^{\prime 2}}{2 \Omega \xi^{4}}-\frac{\xi^{\prime}}{2 \xi^{3}}+\frac{\xi^{\prime}}{2 \xi^{7}}\right]^{\frac{1}{2}},
$$

$$
\Delta p=\left[\frac{1}{2}\left(1-\frac{\omega \xi^{\prime}}{\Omega \xi^{5}}\right) \sqrt{\frac{\xi^{2}-\xi^{-2}}{\xi^{2}-\xi^{\prime 2}}}+\frac{\omega}{2 \Omega \xi^{6}}\right]^{\frac{1}{2}} .
$$

Near the critical point, the critical behavior gives

$$
\Delta x \propto f(\lambda)|\epsilon|^{-1 / 4}, \quad \Delta p \propto f^{-1}(\lambda)|\epsilon|^{1 / 4} .
$$

For $p$-type superradiant phase, $\Delta x$ and $\Delta p$ are obtained via the ground state $\psi_{2}\left(x, \alpha_{2}\right)$.

$$
\Delta x=\left[\frac{1}{2}\left(1-\frac{\omega \xi}{\Omega \xi^{\prime}}\right) \sqrt{\frac{\xi^{\prime 2}-\xi^{\prime-2}}{\xi^{\prime 2}-\xi^{2}}}+\frac{\omega}{2 \Omega \xi^{\prime 6}}\right]^{\frac{1}{2}},
$$

$\Delta p=\left[\frac{1}{2}\left(1-\frac{\omega \xi}{\Omega \xi^{\prime 5}}\right) \sqrt{\frac{\xi^{\prime 2}-\xi^{2}}{\xi^{\prime 2}-\xi^{\prime-2}}}+\frac{\omega \xi^{2}}{2 \Omega \xi^{\prime 4}}-\frac{\xi}{2 \xi^{\prime 3}}+\frac{\xi}{2 \xi^{\prime 7}}\right]^{\frac{1}{2}}$.

Near the critical point, the critical behavior gives

$$
\Delta x \propto f^{-1}(\lambda)|\epsilon|^{1 / 4}, \quad \Delta p \propto f(\lambda)|\epsilon|^{-1 / 4} .
$$

In the $x$-type superradiant phase, $\Delta x$ acts as the diverging length scale, while in the $p$-type superradiant phase, $\Delta p$ is the diverging length scale. According to Eq. (29), the diverging length scale gives the critical exponent $v=1 / 4$ and the dynamical critical exponent $z=2$.

\section{B. Numerical scalings}

Below we show how to numerically extract the KibbleZurek scalings from the non-equilibrium dynamics. We perform the numerical simulations based on the Hamiltonian (2). To study the non-equilibrium dynamics, we prepare the initial ground state deeply in the normal phase, in order to drive the system cross the superradiant phase transition, the coupling strength $\tilde{g}$ is linearly quenched according to

$$
\tilde{g}(t)=\tilde{g}_{c}\left(1-t / \tau_{Q}\right),
$$

where $\tilde{g}_{c}$ is the critical point, and $\tau_{Q}$ is the quench time. The typical total density distributions for different quench times are shown in Fig. 3. When the system is quenched at a fast rate (which respond to small $\tau_{Q}$ ), the state may remain the information of the normal phase even in the deep superradiant region, see Fig. 3(a). However, the state evolves more adiabatic as the quench time becomes larger, in which the oscillation amplitude of the state becomes smaller, see Fig. 3(c). When $\tau_{Q} \rightarrow \infty$, the quench dynamic returns to the equilibrium case, see the Fig. 1(a). Base on aforementioned description, the phonon number $n_{c}=\left\langle\omega\left(p^{2}+x^{2}\right) / 2\right\rangle$ serves as the order parameter. When the coupling strength $\tilde{g}$ is quenched across the phase transition $\tilde{g}_{c}$, the time-evolution of $n_{c}$ 


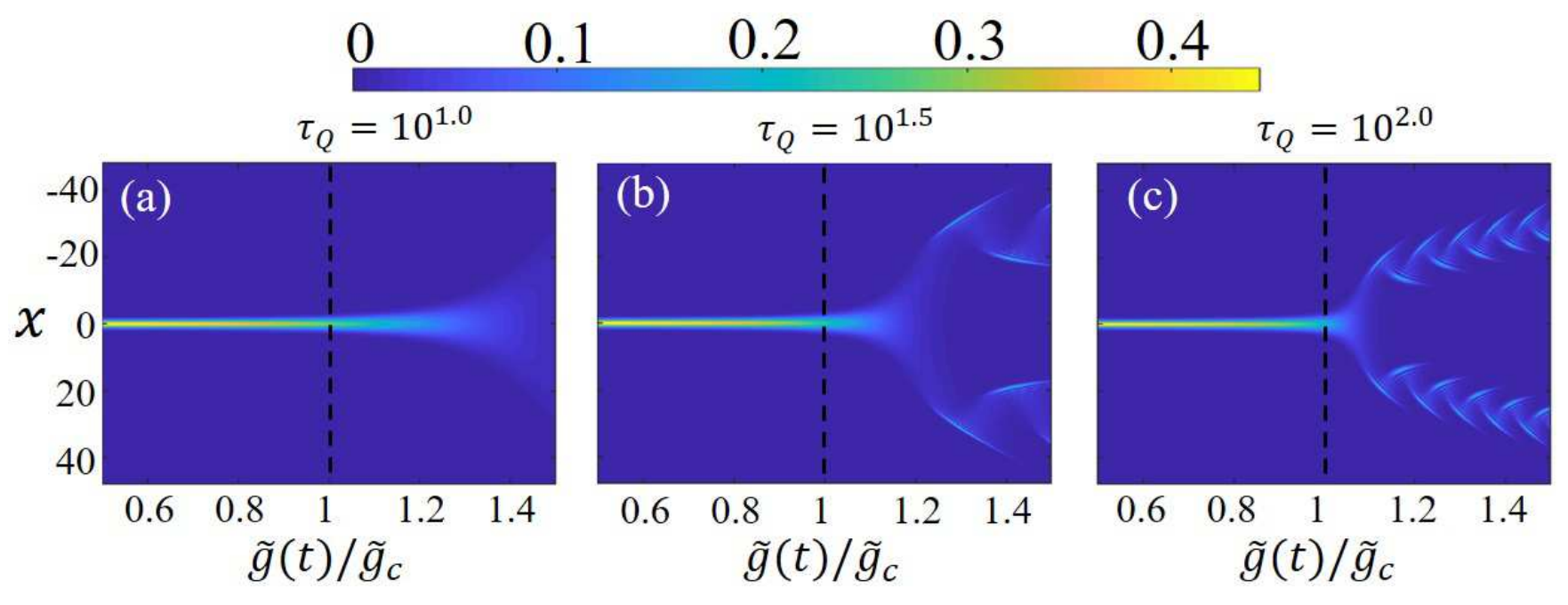

FIG. 3. (Color online) The time-evolution of density distribution for different quench times $\tau_{Q}$. When the system is quenched from normal to superradiant phases at a finite $\tau_{Q}$, due to the vanishing excitation gap at the critical point, the system can't adiabatically across the critical point and the wave-packet spreads due to the appearance of excitations. The parameters are chosen as $L=96, \omega=1, \Omega=1000, \lambda=1$ and $\tau_{Q}=10^{1.0}, 10^{1.5}, 10^{2.0}$.

are shown in Fig. 4(a). In the case of equilibrium phase transition, the phonon number becomes non-zeros when the system sweeps through the critical point. However, in the case of the non-equilibrium dynamics, the phonon number delays to increase until the system crosses the freeze time $\hat{t}$, where the state restarts to evolve.
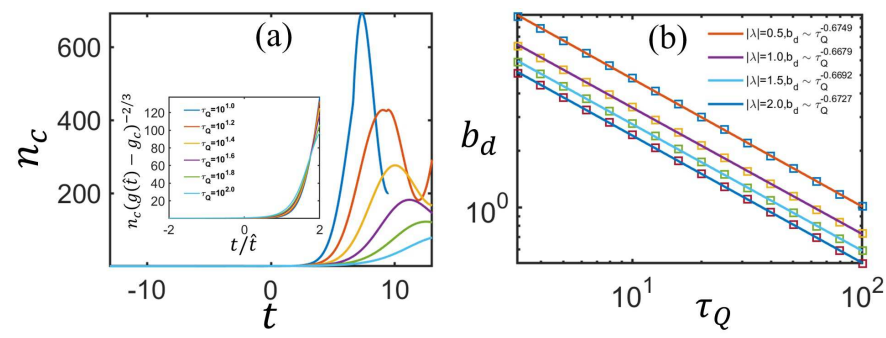

FIG. 4. (Color online) (a) The time evolution of the phonon number $n_{c}$ for different quench times, the inset shows the universal behavior in the rescaled time. (b) The universal scaling of the phase transition delay $b_{d}$ with respect to the quench time $\tau_{Q}$ for different $\lambda$.

To study the phase transition delay $b_{d}$, we define the $b_{d}$ as

$$
b_{d} \sim|\epsilon| \sim\left|\tilde{g}(\hat{t})-\tilde{g}_{c}\right| \sim \tau_{Q}^{-\frac{1}{1+v z}},
$$

where $\hat{t}$ is the freeze time. In our calculation, $\hat{t}$ is determined when the phonon number $n_{c}$ reaches at fixed value $n_{c}^{f i x}$. According to the KZM, the instantaneous state freezes at $-\hat{t}$ but with global phase evolution during the impulse region. Hence, the order parameter remains zero in the first adiabatic region and the impulse region. When the system crosses over the freeze time $\hat{t}$, the instantaneous state restarts to evolve again, but the states at this moment are no longer the eigenstates of the Hamiltonian. Therefore, the order parameter becomes nonzero after the freeze time $\hat{t}$. We determine the freeze time $\hat{t}$ when the phonon number $n_{c}$ satisfies $n_{c}^{f i x}=5$. In Fig. 4(b), we show the universal scaling of the the phase transition delay $b_{d}$ with respect to the quench time $\tau_{Q}$, the numerical scalings for different $\lambda$ are well consistent with the analytical result $b_{d} \sim \tau_{Q}^{-\frac{1}{1+v z}} \sim \tau_{Q}^{-2 / 3}$.
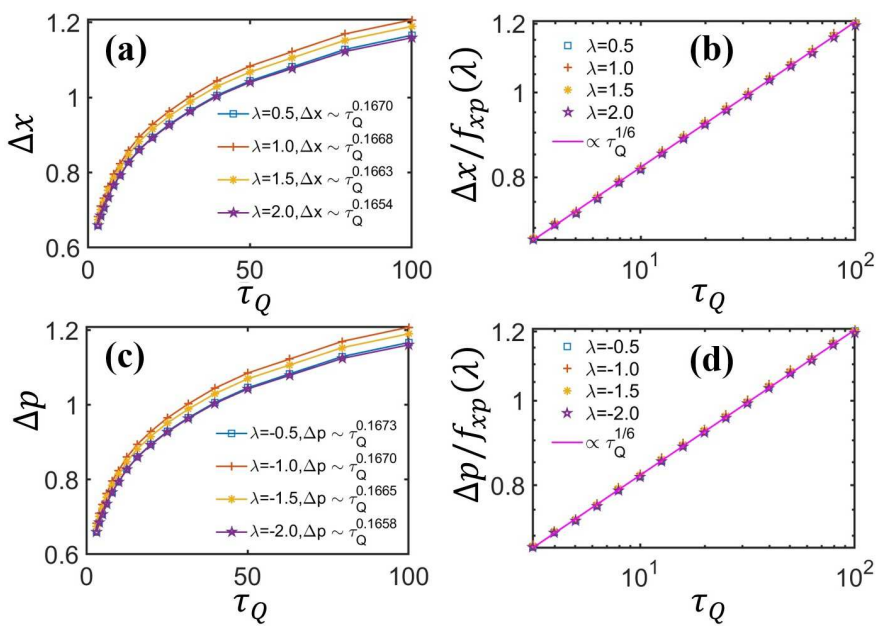

FIG. 5. (Color online) (a,c) Universal scalings of the diverging length scale $\Delta x$ and $\Delta p$ with respect to the quench time $\tau_{Q}$ for different $\lambda$. (b,d) Universal scalings of the rescaled diverging length scale in the logarithmic coordinate.

Here, we treat the position variance $\Delta x$ (or the momentum variance $\Delta p$ ) as the diverging length scale when $\lambda>0$ (or $\lambda<0)$. In Fig. [5 $(a, b)$, we demonstrate the universal scaling of the $\Delta x$ with respect to the quench time $\tau_{Q}$. The power laws are well agree with the analytical 


\begin{tabular}{|l|l|l|l|l|}
\hline Anisotropic ratio $\lambda$ & -0.5 & -1.0 & -1.5 & -2 \\
\hline $\mathrm{z}$ (Critical exponent $)$ & 1.994 & 1.989 & 2.017 & 2.009 \\
\hline$\nu$ (Critical exponent $)$ & 0.2511 & 0.2501 & 0.2507 & 0.2486 \\
\hline Anisotropic ratio $\lambda$ & 0.5 & 1.0 & 1.5 & 2 \\
\hline $\mathrm{z}$ (Critical exponent $)$ & 1.998 & 1.991 & 2.019 & 2.013 \\
\hline$\nu$ (Critical exponent $)$ & 0.2506 & 0.2497 & 0.2504 & 0.2480 \\
\hline
\end{tabular}

TABLE I. The numerical critical exponents $(z, \nu)$ of the anisotropic QRM for different $\lambda$, which are well consistent with the analytical ones $(z=2, \nu=1 / 4)$.

result $\Delta x \sim \tau_{Q}^{\frac{v}{1+v z}} \sim \tau_{Q}^{1 / 6}$. In the case of $\lambda<0$, the momentum variance $\Delta p$ serves as the diverging length scale. Similarly, $\Delta p$ shows universal scaling with respect to the quench time $\tau_{Q}$ as shown in Fig $5(\mathrm{c}, \mathrm{d})$, the power law is well consistent with the analytical result $\Delta p \sim \tau_{Q}^{\frac{v}{1+v z}} \sim \tau_{Q}^{1 / 6}$. Combing the scalings of the phase transition delay and the diverging length scale, we finally give the numerical critical exponents of anisotropic QRM for different $\lambda$ in TABLE. I]

\section{SUMMARY AND DISCUSSIONS}

In summary, we have investigated the non-equilibrium dynamics across a normal-to-superradiant phase transition in the anisotropic QRM. Through performing the SW transformation, the Hamiltonian can be diagonalized and so that the ground states and their excitation gaps can be analytically obtained. By analyzing the excitation gap and the diverging length scale, we give the critical exponents $(z=2, \nu=1 / 4)$. Meanwhile, we also simulate the real-time slow dynamics across the normal-to-superradiant phase transition. To extract the critical exponents, we study the phase transition delay and diverging length scale near the critical point, which show universal scalings with respect to the quench time. By introducing position and momentum operators, we clearly show the spontaneous symmetry breaking in the anisotropic QRM, which manifests the total density distribution spontaneously varies from a singlepeak to double-peak shape. Moreover, we reveal that the anisotropic QRM shares the same universal class (i.e. the identical critical exponents) in spite of the anisotropic ratio.

It is possible to realize the QRM in the ultrastrong coupling regime and the deep strong coupling regime via superconducting circuits [58 60], cold atoms [61] and trapped ions [33, 62]. The realization of the anisotropic QRM is more challengeable, some attempts have been proposed via quantum well [63, 64], circuit QED systems [65, 66], superconducting flux qubits [54]. The ratio between the atomic transition frequency $\Omega$ and the phonon field frequency $\omega$ can be tuned by adjusting the frequency detuning of the time-dependent magnetic fields, the qubits frequency and the LC oscillator frequency. The coupling interaction strength $g$ and the anisotropic ratio $\lambda$ can be tuned by adjusting the phase of the time-dependent magnetic fields.

\section{ACKNOWLEDGMENTS}

This work is supported by the Key-Area Research and Development Program of GuangDong Province under Grants No. 2019B030330001, the National Natural Science Foundation of China (NNSFC) under Grants No. 11874434 and No. 11574405, and the Science and Technology Program of Guangzhou (China) under Grants No. 201904020024.
[1] S. Sachdev, Quantum Phase Transitions 1st edn (Cambridge University Press, 2011).

[2] M. Morikawa, "Cosmological Inflation as a Quantum Phase Transition," Progr. Theoret. Phys. 93, 685 (1995).

[3] T. W. B. Kibble, "Some Implications of a Cosmological Phase Transition," Phys. Rep. 67, 183 (1980).

[4] T. W. B. Kibble, "Topology of Cosmic Domains and Strings," J. Phys. A: Math. Gen. 9, 1387 (1976).

[5] C. Lee, "Universality and Anomalous Mean-Field Breakdown of Symmetry-Breaking Transitions in a Coupled Two-Component Bose-Einstein Condensate," Phys. Rev. Lett. 102, 070401 (2009).

[6] J. Sabbatini, W. H. Zurek, and M. J. Davis, "Phase Separation and Pattern Formation in a Binary Bose-Einstein Condensate," Phys. Rev. Lett. 107, 230402 (2011).

[7] J. Sabbatini, W. H. Zurek, and M. J. Davis, "Causality and Defect Formation in the Dynamics of an Engineered Quantum Phase Transition in a Coupled Binary BoseEinstein Condensate," New. J. Phys. 14, 095030 (2012).

[8] T. Świslocki, E. Witkowska, J. Dziarmaga, and M. Ma- tuszewski, "Double Universality of a Quantum Phase Transition in Spinor Condensates: Modification of the Kibble-Zurek Mechanism by a Conservation Law," Phys. Rev. Lett. 110, 045303 (2013).

[9] J. Hofmann, S. S. Natu, and S. D. Sarma, "Coarsening Dynamics of Binary Bose Condensates," Phys. Rev. Lett. 113, 095702 (2014).

[10] S.-Y. Wu, Y.-G. Ke, J.-H. Huang, and C Lee, "KibbleZurek Scalings of Continuous Magnetic Phase Transitions in Spin-1 Spin-Orbit-Coupled Bose-Einstein Condensates," Phys. Rev. A. 95, 063606 (2017).

[11] J. Xu, S.-Y. Wu, X.-Z. Qin, J.-H. Huang, Y.-G. Ke, H.H. Zhong, and C. Lee, "Kibble-Zurek Dynamics in an Array of Coupled Binary Bose Condensates," Europhys. Lett. 113, 50003 (2016).

[12] N. Navon, A. L. Gaunt, R. P. Smith, and Z. Hadzibabic, "Critical Dynamics of Spontaneous Symmetry Breaking in a Homogeneous Bose Gas," Science 347, 167 (2015).

[13] Q. Z. Ye, S. Y. Wu, X. D. Jiang, and C. H. Lee, "Universal Dynamics of Zero-Momentum to Plane-Wave Transi- 
tion in Spin-Orbit Coupled Bose-Einstein Condensates," J. Stat. Mech. , 053110 (2018).

[14] X. D. Jiang, S. Y. Wu, Q. Z. Ye, and C. H. Lee, "Universality of Miscible-Immiscible Phase Separation Dynamics in Two-Component Bose-Einstein Condensates," New J. Phys. 21, 023014 (2019).

[15] C. N. Weiler, T. W. Neely, D. R. Scherer, A. S. Bradley, M. J. Davis, and B. P. Anderson, "Spontaneous Vortices in the Formation of Bose-Einstein Condensates," Nature 455, 948 (2008).

[16] S. W. Su, S. C. Gou, A. Bradley, O. Fialko, and J. Brand, "Kibble-Zurek Scaling and its Breakdown for Spontaneous Generation of Josephson Vortices in Bose-Einstein Condensates," Phys. Rev. Lett. 110, 215302 (2013).

[17] S.-Y Wu, X.-Z. Qin, J. Xu, and C Lee, "Universal Spatiotemporal Dynamics of Spontaneous Superfluidity Breakdown in the Presence of Synthetic Gauge Fields," Phys. Rev. A. 94, 043606 (2016).

[18] B. Damski and W. H. Zurek, "Soliton Creation During a Bose-Einstein Condensation," Phys. Rev. Lett. 104, 160404 (2010).

[19] E. Witkowska, P. Deuar, M. Gajda, and K. Rzażewski, "Solitons as the Early Stage of Quasicondensate Formation during Evaporative Cooling," Phys. Rev. Lett. 106, 135301 (2011).

[20] W. H. Zurek, "Causality in Condensates: Gray Solitons as Relics of BEC Formation," Phys. Rev. Lett. 102, 105702 (2009).

[21] W. H. Zurek, "Cosmological Experiments in Superfluid Helium?" Nature 317, 505 (1985).

[22] W. H. Zurek, "Cosmological Experiments in Condensed Matter Systems," Phys. Rep. 276, 177 (1996).

[23] J. Dziarmaga, "Dynamics of a Quantum Phase Transition and Relaxation to a Steady State," Ads. Phys. 59, 1063-1189 (2000).

[24] A. Polkovnikov, K. Sengupta, A. Silva, and M. Vengalattore, "Nonequilibrium Dynamics of Closed Interacting Quantum Systems," Rev. Mod. Phys. 83, 863 (2011).

[25] I. Bloch, J. Dalibard, and W. Zwerger, "Many-Body Physics with Ultracold Gases," Rev. Mod. Phys. 80, 885 (2008).

[26] V. M. H. Ruutu, V. B. Eltsov, A. J. Gill, and T. W. B. Kibble, "Vortex Formation in Neutron-Irradiated Superfluid ${ }^{3} \mathrm{He}$ as an Analogue of Cosmological Defect Formation," Nature 382, 334 (1996).

[27] C. Bäuerle, Y. M. Bunkov, and S. N. Fisher, "Laboratory Simulation of Cosmic String Formation in the Early Universe Using Superfluid ${ }^{3}$ He," Nature 382, 1332 (1996).

[28] R. Monaco, J. Mygind, R. J. Rivers, and V. P. Koshelets, "Spontaneous Fluxoid Formation in Superconducting Loops," Phys. Rev. B. 80, 180501 (2009).

[29] A. del Campo, G. De. Chiara, G. Morigi, M. B. Plenio, and A. Retzker, "Structural Defects in Ion Chains by Quenching the External Potential: The Inhomogeneous Kibble-Zurek Mechanism," Phys. Rev. Lett. 105, 075701 (2010).

[30] S. Ulm et al, "Observation of the Kibble-Zurek Scaling Law for Defect Formation in Ion Crystals," Nat. Commum. 4, 2290 (2013).

[31] K. Pyka et al, "Topological Defect Formation and Spontaneous Symmetry Breaking in Ion Coulomb Crystals," Nat. Commum. 4, 2291 (2013).

[32] S. Ejtemaee and P. C. Haljan, "Spontaneous Nucleation and Dynamics of Kink Defects in Zigzag Arrays of Trapped Ions," Phys. Rev. A. 87, 051401 (2013).

[33] D. S. Lv, S. M. An, Z. Y. Liu, J. N. Zhang, J. S. Pedernales, L. Lamata, E. Solano, and K. Kim, "Quantum Simulation of the Quantum Rabi Model in a Trapped Ion," Phys. Rev. X 8, 021027 (2018).

[34] G. Lamporesi, S. Donadello, S. Serafini, F. Dalfovo, and G. Ferrari, "Spontaneous Creation of Kibble-Zurek Solitons in a Bose-Einstein Condensate," Nat. Phys. 9, 656 (2013).

[35] M. Anquez, B. A. Robbins, H. M Bharath, M. Boguslawski, T. M. Hoang, and M. S. Chapman, "Quantum Kibble-Zurek Mechanism in a Spin-1 Bose-Einstein Condensate," Phys. Rev. Lett. 116, 155301 (2016).

[36] L. W. Clark, L. Feng, and C. Chin, "Universal SpaceTime Scaling Symmetry in the Dynamics of Bosons Across a Quantum Phase Transition," Science 354, 606 (2016).

[37] L. Feng, L. W. Clark, A. Gaj, and C. Chin, "Coherent Inflationary Dynamics for Bose-Einstein Condensates Crossing a Quantum Critical Point," Nat. Phys. 14, 269 (2018).

[38] P. Forn-Diaz, L. Lamata, E. Rico, J. Kono, and E. Solano, "Ultrastrong Coupling Regimes of LightMatter Interaction," Rev. Mod. Phys. 91, 025005 (2019).

[39] A. F. Kockum, A. Miranowicz, S. De Liberato, S. Savasta, and F. Nori, "Ultrastrong Coupling Between Light and Matter," Nat. Rev. Phys. 1, 19 (2019).

[40] I. I. Rabi, "On the Process of Space Quantization," Phys. Rev. 49, 324 (1936).

[41] I. I. Rabi, "Syace Quantization in a Gyrating Magnetic Field," Phys. Rev. 51, 652 (1937).

[42] H. H. Zhong, Q. T. Xie, M. T. Batchelor, and C. H. Lee, "Analytical Eigenstates for the Quantum Rabi Model," J. Phys. A: Math. Theor. 46, 415302 (2013).

[43] H. H. Zhong, Q. T. Xie, X. W. Guan, M. T. Batchelor, K. L. Gao, and C. H. Lee, "Analytical Energy Spectrum for Hybrid Mechanical Systems," J. Phys. A: Math. Theor. 47, 045301 (2014).

[44] Q. T. Xie, H. H. Zhong, M. T. Batchelor, and C. H. Lee, "The Quantum Rabi Model: Solution and Dynamics," J. Phys. A: Math. Theor. 50, 113001 (2017).

[45] S. Ashhab, "Superradiance Transition in a System with a Single Qubit and a Single Oscillator," Phys. Rev. A. 87, 013826 (2013).

[46] R. F. Bishop and N. J. Davidson, "Application of the Coupled Cluster Method to the Jaynes-Cummings Model Without the Rotating-Wave Approximation," Phys. Rev. A. 54, 4657 (1996).

[47] J. Larson and E. K. Irish, "Some Remarks on 'Superradiant' Phase Transitions in Light-Matter Systems," J. Phys. A: Math. Theor. 50, 174002 (2017).

[48] M. J. Hwang and M. S. Choi, "Variational Study of a Two-Level System Coupled to a Harmonic Oscillator in an Ultrastrong-Coupling Regime," Phys. Rev. A. 82, 025802 (2010).

[49] M. J. Hwang, P. Rabl, and M. B. Plenio, "Dissipative Phase Transition in the Open Quantum Rabi Model," Phys. Rev. A. 97, 013825 (2018).

[50] R. Puebla, M. J. Hwang, J. Casanova, and M. B. Plenio, "Probing the Dynamics of a Superradiant Quantum Phase Transition with a Single Trapped Ion," Phys. Rev. Lett. 1198, 073001 (2017).

[51] M. J. Hwang, R. Puebla, and M. B. Plenio, "Quantum Phase Transition and Universal Dynamics in the Rabi 
Model," Phys. Rev. Lett. 115, 180404 (2015).

[52] Q. T. Xie, S. Cui, J. P. Cao, L. G. Amico, and H. Fan, "Anisotropic Rabi Model," Phys. Rev. X. 4, 021046 (2014).

[53] Y. Y. Zhang and X. Y. Chen, "Analytical Solutions by Squeezing to the Anisotropic Rabi Model in the Nonperturbative Deep-Strong-Coupling Regime," Phys. Rev. A. 96, 063821 (2017).

[54] Y. M. Wang, W. L. You, M. X. Liu, Y. L. Dong, H. G. Luo, G. Romero, and J. Q. You, "Quantum Criticality and State Engineering in the Simulated Anisotropic Quantum Rabi Model," New J. Phys. 20, 053061 (2018).

[55] M.-X. Liu, S. Chesi, Z.-J. Ying, X.-S. Chen, H.-G. Luo, and H.-Q. Lin, "Universal Scaling and Critical Exponents of the Anisotropic Quantum Rabi Model," Phys. Rev. Lett. 119, 220601 (2017).

[56] Z.-J. Ying, M.-X. Liu, H.-G. Luo, H.-Q. Lin, and J. Q. You, "Ground-State Phase Diagram of the Quantum Rabi Model," Phys. Rev. A. 92, 053823 (2015).

[57] M. J. Hwang and M. B. Plenio, "Quantum Phase Transition in the Finite Jaynes-Cummings Lattice Systems," Phys. Rev. Lett. 117, 123602 (2016).

[58] N.K. Langford, R. Sagastizabal, M. Kounalakis, C. Dickel, A. Bruno, F. Luthi, D.J. Thoen, A. Endo, and L. DiCarlo, "Experimentally Simulating the Dynamics of Quantum Light and Matter at Deep-Strong Coupling," Nat. Commum. 8, 1715 (2017).

[59] J. Braumüller, M. Marthaler, A. Schneider, A. Stehli,
H. Rotzinger M. Weides, and A. V. Ustinov, "Analog Quantum Simulation of the Rabi Model in the UltraStrong Coupling Regime," Nat. Commum. 8, 779 (2017).

[60] C. Leroux, L. C. G. Govia, and A. A. Clerk, "Enhancing Cavity Quantum Electrodynamics via Antisqueezing: Synthetic Ultrastrong Coupling," Phy. Rev. Lett. 120, 093602 (2018).

[61] S. Felicetti, G. Romero, E. Solano, and C. Sabín, "Quantum Rabi Model in a Superfluid Bose-Einstein Condensate," Phys. Rev. A 96, 033839 (2017).

[62] J. S. Pedernales, I. Lizuain, S. Felicetti, G. Romero, L. Lamata, and E. Solano, "Quantum Rabi Model with Trapped Ions," Sci. Rep. 5, 15472 (2015).

[63] J. Schliemann, J. C. Egues, and D. Loss, "Variational Study of the $\nu=1$ Quantum Hall Ferromagnet in the Presence of Spin-Orbit interaction," Phys. Rev. B 67, 085302 (2003).

[64] Z. H. Wang, Q. Zheng, X. G. Wang, and Y. Li, "The Energy-Level Crossing Behavior and Quantum Fisher Information in a Quantum Well with Spin-Orbit Coupling," Sci. Rep. 6, 22347 (2016).

[65] A. Baksic and C. Ciuti, "Controlling Discrete and Continuous Symmetries in "Superradiant" Phase Transitions with Circuit QED Systems," Phys. Rev. Lett. 112, 173601 (2014).

[66] W. J. Yang and X. B. Wang, "Ultrastrong-Coupling Quantum-Phase-Transition Phenomena in a Few-Qubit Circuit QED System," Phys. Rev. A 95, 043823 (2017). 